\title{
MiR-219-5p is Involved in the Proliferation, Migration and Invasion of Oral Cancer Through SoX5 Regulation
}

\section{Ting Zhou}

Kunming Medical University

\section{Ying He}

Yunnan Province Corps Hospital,Chinese People's Armed Police Forces

\section{Xiao-Han Dong}

Kunming Medical University

\section{Bo Chen}

Kunming Medical University

\section{Jun Ton}

Kunming Medical University

\section{Yan-Bin Xiyang}

Kunming Medical University

Rui Mao ( $\triangle$ maorui@kmmu.edu.cn )

Kunming Medical University https://orcid.org/0000-0002-6490-8291

Primary research

Keywords: oral cancer, miR-219-5p, SOX5, proliferation, migration, invasion

Posted Date: June 8th, 2021

DOl: https://doi.org/10.21203/rs.3.rs-574925/v1

License: (9) This work is licensed under a Creative Commons Attribution 4.0 International License. Read Full License 


\section{Abstract}

Purpose Oral cancer has the characteristics of rapid progression, wide invasion and poor prognosis, which induces higher mortality in the patients. At present, there are about 300 thousand new cases of oral cell carcinoma worldwide. Particularly, the incidence rate of oral cancer in China is relatively high. Therefore, it urgently needs to understand the pathogenesis of oral cancer and molecular mechanisms underlying. Abnormal regulation of miR-219-5p is present in various types of cancer. However, the relationship between miR-219-5p and its targets in oral cancer has not been well evaluated.

Methods Western blotting and Quantitative RT-PCR were used to detect the expression of SOX5 in oral cancer tissues.Migration ,cell proliferation,and invasion were detected using CCK8 assay,Conlony formation assay and Transwell assays.The interaction between SOX5 and miR-219-5p and oral cancer was confirmed using dual-luciferase reporter assays.

Result This study aims to investigate the possible roles of miR-219-5p and its potential target gene, SOX5, in the progress of oral cancer. Our data showed that the high miR-219-5p and low SOX5 expression levels were associated with improved survival rates in patients. miR-219-5p level was negatively correlated with the expression of SOX5. Genetic analysis and luciferase assay revealed that the miR-291-5p regulated SOX5 expression by targeting the 3'-UTR region of SOX 5 mRNA. Functionally, we confirmed that miR-219$5 p$ mimics inhibited SOX 5 expression and suppressed the proliferation, colony formation ability, invasion and migration of oral cancer cells, SCC4 and SCC9. In contrast, inhibition of miR-219-5p increased SOX5 levels and promoted the vitality and mobility of oral cancer cells. Furthermore, special siRNA targeting SOX5 partially neutralized the effects of miR-219-5p inhibitor.

Conclusions This study demonstrates that miR-219-5p may inhibit the proliferation, migration and invasion of oral cancer by targeting the expression of SOX5, which provided novel candidates for clinic prognosis and/or therapy.

\section{Introduction}

Head and neck cancer is the sixth most common cancer in humans, accounting for about $3 \%$ of all cancers. Among all head and neck cancers, $48 \%$ of cancers occur in the oral cavity, which is collectively referred to as oral cancer[1]. Despite advances in the treatment of oral cancer, patients with oral cancer has a very poor prognosis and low survival rate. Therefore, it is expected to make a breakthrough in the early diagnosis and treatment of oral cancer by integrating multiple molecular targets for prediction or treatment based on the further researches.

Small noncoding micro-RNAs (miRNAs) are a class of single-stranded RNA molecules with length of 1725 nucleotides, which play an important role in the regulation of posttranscriptional gene expression. After mRNA is produced by gene transcription, miRNA can interact with the 3'- untranslated region (3'UTR) of target messenger RNA (mRNA) to induce the direct degradation of target mRNA and subsequently inhibit the expression of target protein[2囚3]. Current studies have found that more than $30 \%$ 
of human genes are regulated by miRNA, and the same miRNA can regulate the degradation of hundreds of mRNAs[4]MiRNA is involved in cell proliferation and differentiation, development, apoptosis, inflammation, as well as tumor genesis and metastasis by regulating the expression of targeted genes. Studies have shown that miRNAs can act as proto-oncogenes or tumor suppressors to regulate the signaling pathways involved in gene expression and the proteins encoded by the modified genes. Previous reports have shown that miRNAs are also involved in the occurrence and development of oral cancer. Further in vitro studies find that miR-219-5p inhibits the proliferation, migration and invasion of various cancer cells[586]. However, the expression and roles of miR-219-5p in oral cancer are still unclear.

SOX5 (SRY-related high-mobility-group box 5) is a member of the SOX transcription factor family. The human SOX 5 protein has two isomers, the longer and shorter SOX 5 isomer. The former encodes 763 amino acids, which is expressed in the heart, brain, liver, lung, spleen, and testes. The latter isomer, which encodes 750 amino acids, is expressed specifically only in testes[7 78 ]. Recent studies demonstrate that SOX5 is involved in promoting the development and metastasis of nasopharyngeal carcinoma, pituitary

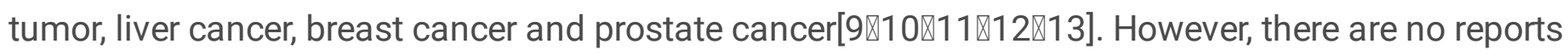
about the expression of SOX5 in oral cancer and its role in the development and metastasis of oral cancer. Therefore, this study on the expression changes of miR-219-5p and its potential targets in oral cancer will help us to understand the molecular mechanism of the occurrence and progress of oral cancer.

\section{Methods}

\section{Clinical samples collection}

Fifty-six pairs of cancer tumor tissues and adjacent normal tissues were obtained from patients with oral cancer who received surgery at the Affiliated stomatological hospital of Kunming Medical University between January 2014 and December 2015 . Specimens were immediately stored at $-80^{\circ} \mathrm{C}$ until nucleic acids and proteins were isolated. All the patients recruited were undergoing surgery in the hospital for the first time and had not received anti-tumor treatment prior to surgery.

All procedures performed in the present study involving human participants were conducted in accordance with the 1964 Declaration of Helsinki and its later amendments or comparable ethical standards revised in 2000. The present study was approved by The Medical Ethics Committee of Kunming Medical University (Kunming, China). All the patents involved in this study were required the informed consents.

\section{Cell preparation}

The human tongue squamous carcinoma cells, SCC4 and SCC9, and the human normal skin fibroblast cell line, HF, were purchased from The Cell Bank of Type Culture Collection of The Chinese Academy of Sciences (Shanghai, China). The SCC4 and SCC9 cells were verified by The Cell Bank of Type Culture 
Collection of the Chinese Academy of Sciences. No misidentified and/or contaminated cell lines were used in the present study.

The cells were 12th generations and cultured in the mixture medium, which contained Dulbecco's Modified Eagle's Medium (DMEM)/F12 (ThermoFisher Scientific, Shanghai, China), 2 mM glutamine, 10\% fetal bovine serum, $100 \mathrm{U} / \mathrm{ml}$ penicillin and $100 \mathrm{mg} / \mathrm{ml}$ streptomycin (ThermoFisher Scientific, Shanghai, China). The culture conditions were $37^{\circ} \mathrm{C}$ with a humidified atmosphere containing $5 \% \mathrm{CO}_{2}$.

\section{Cell treatment}

The SCC4 and SCC9 cell lines were transiently transfected with either miR-219-5p mimics $(1.5 \mu \mathrm{l}, 3 \mu \mathrm{M})$ or miR-219-5p inhibitor $(5 \mu \mathrm{l}, 2.5 \mu \mathrm{M})$ or the matched control using Lipofectamine 3000 reagent (Invitrogen; Thermo Fisher Scientific, Inc.). The miR-219-5p mimics, miR-219-5p inhibitor, miRNA mimics negative control (miR-NC) and scrambled siRNA negative control (miR-219-5p inhibitor NC) were provided by Guangzhou RiboBio Co., Ltd. The sequences of the miRNAs used are described as: miR-219-5p mimics: UGAUUGUCCAAACGCAAUUCU; miR-NC: UGGAUUCCUUAACUUCCCAA; miR-219-5p inhibitor: UUCAACCAGGAAAAGUUGGGAAU; miR-219 inhibitor NC: AUGGCACGUGUACUCCUACAUAC. The $1 \times 10^{6}$ cells were seeded in a plate and transfected with the reagents described above for $48 \mathrm{~h}$ at $37^{\circ} \mathrm{C}$ as recommended by the manufacturer's protocol. Subsequently, the treated cells were harvested for further analyses, including Reverse transcription-quantitative PCR (RT-qPCR), Western blot, Cell proliferation, colony formation assay and migration assays.

pcDNA3.1 (+) plasmid or siRNAs was purchased to up-regulate or down-regulate the expression levels SOX5 in oral cancer cells, respectively. Briefly, the full-length ORF of SOX5 was amplified by PCR, and then the cDNA was connected with pcDNA3.1 (+) plasmid (Invitrogen) by T4 ligase (Takara Bio Inc.). DNA sequencing was used to verify whether the recombinant plasmid (pcDNA3.1-SOX5) was correct. The constructed pcDNA3.1-SOX5 plasmid was transfected into SCC4 and SCC9 with Lipofectamine 2000 reagent (Invitrogen) according tot the protocol provided by manufacturers. Twenty-four hours post transfection, the expression levels of SOX5 were detected by RT-qPCR and Western blot.

Three specific siRNAs targeting human SOX5 mRNA and control siRNA were designed and synthesized by Sigma-Aldrich. The control and SOX 5 siRNAs were transiently transfected into SCC4 and SCC9 with Lipofectamine 2000 reagent (Invitrogen), respectively. After $24 \mathrm{~h}$ of transfection, the total RNA and protein were extracted from the cells to evaluate the changes in expression levels of SOX5 mRNA or protein by RT-qPCR or Western blot, respectively. Two siRNAs with high efficiency were selected for subsequent experiments.

\section{Verification SOX5 as the target gene of miR-219-5p and the binding regions}

According to the computational algorithms miRMine (http://www.microrna.org), the binding site of miR219-5p in the 3 '- UTR of SOX5 mRNA was predicted to be 5 ' - GGACCAAA -3 '. Subsequently, the corresponding mutation site sequence was designed as 5' - GCAGGCCA -3 '. Luciferase reporter assays 
were performed to validate the miR-219-5p target gene SOX5 in oral cancer cells. The wild-type (wt) and mutant (mut) sequences were inserted into pmirglo vector (Promega company) by molecular cloning. For luciferase activity assay, miR-219-5p mimic or inhibitor were transfected into oral cancer cells with wtSOX 5 and mut-SOX5 reporter plasmid, respectively. After $48 \mathrm{~h}$ of transfection, luciferase activities of both firefly and renilla were measured by using a Dual-Luciferase Reporter assay system (Promega, Madison, $\mathrm{WI}$ ). Renilla luciferase activities were evaluated as an internal control. Firefly luciferase activity was normalized to that of Renilla luciferase activity. All experiments were performed three times in triplicate.

\section{CCK-8 Assay}

$1 \times 10^{4}$ SCC 4 or SCC9 cells were inoculated into one well of 96 well plate. The constructed plasmids were transiently transfected with Lipofectamine rnaimax reagent (ThermoFisher Scientific, Shanghai, China) for 48 hours. $10 \mu$ l CCK-8 solution (Sigma Aldrich company) was added to each well and incubated in the cell incubator at $37^{\circ} \mathrm{C}$ for 1.5 hours. The plate reading was analyzed at $490 \mathrm{~nm}$ absorbance.

\section{Colony formation assay}

The constructed plasmid was transiently transfected into oral cancer cells SCC4 and SCC9 with Lipofectamine 2000 reagent. After 48 hours, the cells were seeded on a 6-well culture plate covered with $0.6 \%$ agarose for culture. After 14 days of culture, fixed cell colonies were stained with crystal violet (Sigma Aldrich company), and the number of cell colonies were observed under microscope.

\section{RT-qPCR}

Samples from the clinical tissues or from human tongue squamous carcinoma cells were used to assess the relative expression levels of miR-219-5p and SOX5 by RT-qPCR as preciously described[14]. Briefly, TRIzoI ${ }^{\circledR}$ (Invitrogen; Thermo Fisher Scientific, Inc.) and an miRNeasy mini kit (Qiagen AB) were purchased for extracting the total RNA. According to the manufacturer's protocol, the All-in-OneTM miRNA quantitative RT-PCR Detection kit (GeneCopoeia, Inc.) was employed to the RT-qPCR analysis for miR-2195p. The miRNA RT-PCR primers of hsa-miR-219-5p and U6 (reference gene) were provided by Applied Biosystems; Thermo Fisher Scientific, Inc. U6 was served as a reference gene and the expression levels of the examined samples were normalized to those of U6[15]. The sequences of hsa-miR-219-5p primers are described as: forward: 5'- UAUCCACUUGAUCG- GCCUAC-3', reverse: 5'- GCUCCGCTGUAGACCUA UCUGCA$3^{\prime}$.

Moloney Murine Leukemia Virus Reverse Transcriptase (Takara Bio, Inc.) and 2X Mix SYBR Green I (Takara Bio, Inc.) were employed to evaluate the expression of SOX5 mRNA. Each PCR reaction was composed of two parts, including the initial denaturation at $95^{\circ} \mathrm{C}$ for $3 \mathrm{~min}$ and multiple cycles of amplification ( $36 \mathrm{cycles}$ at $95^{\circ} \mathrm{C}$ for $10 \mathrm{sec}, 56.5^{\circ} \mathrm{C}$ for $20 \mathrm{sec}$, and $75^{\circ} \mathrm{C}$ for $30 \mathrm{sec}$ ), followed by a denaturation step at $95^{\circ} \mathrm{C}$ for $15 \mathrm{sec}$, an annealing step at $65^{\circ} \mathrm{C}$ for $10 \mathrm{sec}$, and a final extension step at $95^{\circ} \mathrm{C}$ for $10 \mathrm{sec}$. GAPDH was used as the reference control. The primers were purchased from Invitrogen (Invitrogen; Thermo Fisher Scientific, Inc. ). The primers sequences were described as follows: GAPDH 
forward, 5'-CGAGATCCCTCCAAAATCAA-3' and reverse, 5'- TTCACACCCATGACGAAC AT-3'; and SOX5

forward, 5'-CGTAACGAGCCTATGCAAT-3' and reverse, 5'-TACCGGAAGCTACAATGCA-3'. The $2^{-\triangle \triangle C q}$ method was performed to evaluate the relative levels of the genes[16].

\section{Western blot analysis}

According to the manufacturer's instruction, the BCA detection kit (Beijing Biotechnology, China) was used to detect the protein concentration. Protein samples $(40 \mathrm{mg}$ ) were separated by $10 \%$ SDS-PAGE and transferred to nitrocellulose membrane (beyotime biotechnology, China). The expression levels of SOX5 protein was assessed. Briefly, the membrane was sealed with $5 \%$ skim milk for two hours, then incubated with primary antibody, SOX5 (1:1000; cat.no.sc-293215;Santa Cruz Biotechnology, Inc.) and GAPDH (1:500; cat. no. sc-47724; Santa cruz Biotechnology, Inc.) overnight at $4^{\circ} \mathrm{C}$. Following the primary antibody incubation, the membranes were washed by $0.01 \mathrm{M}$ PBS buffer solution for three times and incubated with IgG binding protein-HRP (1:2,000; cat. no. sc-516102) secondary antibody at $20-24^{\circ} \mathrm{C}$ for $2 \mathrm{~h}$. The protein signal of cell membrane was observed with enhanced chemiluminescence reagent (EMD Millipore). GAPDH was used as the reference for protein expression.

\section{Migrating and invading assay}

Cells with a density of $1 \times 10^{5} / \mathrm{ml}$ were prepared in serum-free medium, and the cell migration or invading experiment was carried out in the upper chamber. Matrigel solution (BD, Franklin lakes, NJ, USA) was used. After incubation for 48 hours, the cells migrated to the upper membrane surface were physically cleaned / removed. The cells migrated or invaded into the membrane were collected for statistical analysis. The cells were stained with $0.1 \%$ crystal violet and $20 \%$ methanol for 20 min and fixation with $20 \%$ methanol for $30 \mathrm{sec}$ at $20-24^{\circ} \mathrm{C}$. The stained cells were then imaged and counted with ibx3 inverted microscope (Olympus, Tokyo, Japan).

\section{Statistical analysis}

The data were expressed as mean \pm standard deviation (SD). In this study, SPSS software (spssinc., version 21.0, Chicago, IL) was used for analysis. Multivariate logistic regression analysis was used to evaluate the relationship between the expression of miR-219-5p (low or high level), SOX5 (low or high level) and oral cancer. The median expression levels of miR-219-5p or SOX5 were used as the cutoff values[17]. Kaplan-Meier method and log-rank test were performed to evaluate the five-year overall survival (OS) curves of oral cancer patients with different expressional levels of miR-219-5p or SOX5. Significant differences between mRNA and proteins expression levels, cell viability, colony numbers and migration and invasive activities, among multiple groups were determined using ANOVA and Student-Newman-Keuls test. $P<0.05$ was considered to indicate a statistically significant difference.

\section{Results}


The expression changes of SOX5 and miR-219-5p were analyzed in cancer samples and tongue squamous carcinoma cells by RT-qPCR and Western blot. The data showed that the expression levels of SOX 5 mRNA and protein in cancer tissues and cells was significantly increased compared with the matched control cohorts, respectively $(P<0.05$, Fig.1a). On the other hand, after searching various cancer databases, we found that there was no previous study on the expression of miR-219-5p in oral cancer. Therefore, we compared the expression of miR-219-5p in oral cancer and paracancerous tissues using RTqPCR. The results showed that the expression level of miR-219-5p was much lower in oral cancer (vs. adjacent normal tissue, $P<0.05$, Fig.1a).

Similarly, further comparison between the normal human HF and tongue squamous carcinoma cells revealed significant increases in SOX5 and decreases in miR-219-5p expression levels in the SCC 4 and SCC9 cells, compared with that of HF respectively $(P<0.05$, Fig. 1 b).

According to the OS curves analysis, oral cancer patients with high expression of miR-219-5p (cut-off level $0.64 ;>0.64$ high expression) demonstrated a higher rate OS compared with patients with low expression ( $\leq 0.64$, low expression) $\left(\chi^{2}=15.24, P=0.00031\right.$; Fig. $\left.1 \mathrm{c}\right)$. The OS rate of patients with low levels of SOX5 (cut-off level 2.86; $\leq 2.86$, low expression) in tumors was higher compared with high levels of SOX5 (>2.86, high expression) $\left(X^{2}=18.11, P=0.00027\right.$; Fig. 1 d).

These results suggests that the abnormal expressions of miR-219-5p and/or SOX5 may be associated with the occurrence and development of oral cancer.

\section{miR-219-5p regulates the expression of SOX5 in oral cancer cells by targeting 3 '- UTR region}

Since SOX5 and miR-219-5p showed the opposite expressional tendency in oral cancer tissues and cells, we speculated that miR-219-5p might target SOX5 mRNA by specific "seed" region. In order to validate this speculation, miRMine was employed to predict the possible binding site of miR-219-5p in SOX5 mRNA. The results showed that there was indeed a binding site of miR-219-5p on the 3 '- UTR of SOX5 mRNA and SOX5 is a potential target gene of miR-219-5p (Fig.2a).

Transfection of SOX5 3'UTR-wt in combination with miR-219-5p mimic (SOX5 3'UTR-wt+miR-219-5p) significantly reduced the luciferase activity compared with the corresponding control (SOX5 3'UTR$w t+m i R-N C)$. However, in cells transfected with SOX5 3'UTR-mut vector, miR-219-5p did not induce the reduction of luciferase activity in SCC4 nor SCC9 cells $(P<0.05$, Fig.2b).

To further determine whether miR-219-5p regulated the expression levels of SOX5, SCC4 and SCC9 cells were treated with miR-219-5p mimics or inhibitors and subsequently the expression levels of SOX 5 were detected. The results showed that miR-219-5p mimic dramatically suppressed SOX5 expressions, while miR-219-5p inhibitor significantly restored the expression of SOX5 in SCC4 and SCC9 cells $(P<0.05$, Fig.2c, d, e). These results validated that miR-219-5p targeted SOX5 by binding to the specific 3 '- UTR region of SOX5 mRNA. 
Investigate the possible roles of SOX5 in the proliferation, migration and invasion of oral cancer cells

The results showed the mRNA and protein expression levels in SCC4 and SCC9 cells with pcDNA3.1SOX5 treatment were significantly higher than that of the pcDNA3.1 plasmid transfected control group $(P<0.05$, Fig.3a,b).

Following siRNAs (siRNA-1, siRNA-2 and siRNA-3) treatment, the expression levels of either SOX5 mRNA and protein were interfered. Among the three siRNAs fragments, siRNA-1 showed the best interfering effect which induced significant decreases in SOX5 mRNA and protein of SCC4 and SCC9 cells $(P<0.05$, Fig.3c,d). Therefore, siRNA-1 fragment was selected for subsequent assessments.

\section{SOX5 promotes colony formation of SCC4 and SCC9 cells}

In order to observe the number and size of cell colony, the cell colony experiment was carried out. It was observed that the cell colonies in pCDNA3.1-SOX5 treated SCC4 and SCC9 cells significantly increased, compared with that of pcDNA3.1 plasmid treated control cells $(P<0.05)$. By contrast, the colony number in siRNA-SOX5 treated cells was significantly lower than that of scrambled siRNA control treated SCC4 and SCC9 cells $(P<0.05$, Fig.4a,b).

\section{SOX5 enhances the vitality of oral cancer cells}

Treatment with pcDNA3.1-SOX5 significantly promoted proliferation of SCC4 and SCC9 cells (vs. pcDNA3.1 plasmid treated ones, $P<0.05)$, which indicated by CCK-8 assay. The CCK-8 assay also revealed that the optical density in SCC4 and SCC9 cells marked reduced after transfecting with siRNA-SOX5, compared with that of siRNA control treated ones $(P<0.05)$. These results suggested that SOX 5 facilitated the proliferation of oral cancer cells, while SOX5 knockdown impaired the cell viability $(P<0.05$, Fig.4 c).

\section{The effects of SOX5 overexpression and knockdown on the migration and invasion of oral cancer cells}

The migration and invasion OSCC cell numbers with pcDNA3.1-SOX5 transfection were significantly larger than that of pcDNA3.1 plasmid control treated cells $(P<0.05)$. It suggested that the overexpression of SOX5 enhanced the migration and invasion of SCC4 and SCC9 cells. Transwell migration and invasion assay was performed according to BD Bioscience and Sigma-Aldrich kit instructions to determine the effects of SOX5 knockdown on the migration and invasion of oral cancer cells. The results also showed that the cell migration and invasion ability of OSCC cells treated with siRNA-SOX 5 was obviously weakened, compared with that of scramble siRNA-SOX 5 control treated ones $(P<0.05$,Fig.5a,b)

Investigate the possible roles of miR-219-5p in the proliferation, migration and invasion of oral cancer cells

The miR-219-5p mimics or inhibitors were transiently transfected into oral cancer cells SCC4 or SCC9 with Lipofectamine rnaimax reagent. It was observed that the apoptosis rates of SCC4 and SCC9 cells transfected with miR-219-5p mimics were higher than those of cells treated with negative control (NC) $\square$ 
The apoptosis rate of SCC4 and SCC9 cells transfected with miR-219-5p inhibitor was lower than that of NC cells transfected with miR-219-5p inhibitor $(P<0.05$,Fig.6a).

\section{miR-219-5p inhibits colony formation of SCC4 and SCC9 cells}

In order to observe the effect of miR-219-5p on cell colony, the experiment was conducted, and the results showed that the number and size of cells transfected with miR-219-5p mimic were significantly lower than that of miR-NC, while the number and size of cells transfected with miR-219-5p inhibitor were significantly increased, compared with the control group.(compared with NC of each group, there was statistical significance, $P<0.05$,Fig.6b,c).

\section{miR-219-5p inhibits the vitality of oral cancer cells}

The mimics and inhibitors of miR-219-5p were transfected and the the cell viability were determined by CCK8 assays and analyzed. The results showed that the OD490 nm values of cells transfected with miR219-5p mimics was significantly lower than that of the cells treated with miR-NC, while the OD490 nm values of cells transfected with miR-219-5p inhibitors was significantly higher than that of the control group $(P<0.05$, Fig.6d).

\section{The effects of miR-219-5p on the migration and invasion of oral cancer cells.}

The miR-219-5p mimic or inhibitor was transfected into oral cancer cells, SCC4 and SCC9, to detect the effects of miR-219-5p on the migration and invasion of oral cancer cells. The experiment showed that transfection with miR-219-5p mimics significantly reduced the migration and invasion of SCC4 and SCC9 cells, compared with control negative control -treated (miR-NC) cells, respectively. However, miR-219-5p inhibitor treatment significantly promoted the migration and invasion of SCC4 and SCC9 cells $(P<0.05$, Fig.6e,f).

\section{miR-219-5p inhibits the vitality and mobility of oral cancer cells by targeting SOX5}

siRNA-SOX5 transfection effectively reduced the colony numbers $(P<0.05$,Fig.7a), values of optic density 490nm (OD 490nm, indicated by CCK-8 assay; $(P<0.05$,Fig.7c), migrating and invading numbers $(P<0.05$,Fig.8a,b), while pcDNA3.1-S0X5 transfection marked increased the colony numbers $(P<0.05$,Fig.7b) and OD $490 \mathrm{~nm}$ values $(P<0.05$,Fig.7d), and migrating and invading numbers $(P<0.05$,Fig.8a,b) of SCC4 and SCC9 cells. The data also showed that compared with the blank control group, the colony numbers and OD 490nm values, as well as the migration and invading cell numbers of SCC4 and SCC9 cells were significantly increased by transfection with miR-219-5p inhibitor, while those increases were partially neutralized by combined siRNA-SOX5 treatment $(P<0.05$, Fig.7a,c Fig.8a,b).

On the contrary, the colony numbers, values of OD $490 \mathrm{~nm}$, migrating and invading oral cancer cells transfected with miR-219-5p mimics were significantly reduced, compared with the blank control group $(P<0.05$,Fig.7b, d Fig.8a,b). Furthermore, combined transfection with miR-219-5p mimics and pcDNA3.1SOX5 alleviated those suppression in proliferation and migration induced by miR-219-5p mimics, and 
partially restored the colony formation ability, OD 490nm values, as well as migrating and invading capacities of oral cancer cells $(P<0.05$,Fig.7b, d Fig. 8a,b)

\section{Discussion}

The results showed that the expression levels of SOX5 in oral cancer tissues and oral cancer cells were higher than that in paracancerous tissues, while they were lower than that in paracancerous tissues. Oral cancer patients with high expression of miR-219-5p demonstrated a higher OS rate compared with patients with low expression, while the OS rate of patients with low levels of SOX5 in tumors was higher compared with high levels of SOX5. In addition, the expression of SOX5 in oral cancer tissues was negatively correlated with miR-219-5p. Luciferase report assays and in vitro experiments confirmed that SOX 5 was a target gene of miR-219-5p and miR-219-5p regulated the expression levels of SOX5. It also found that SOX5 and miR-219-5p played important roles in the the proliferation, migration and invasion of oral cancer cells. miR-219-5p inhibitor or SOX5 overexpression enhanced cell viability, while miR-219$5 p$ mimics or SOX5 siRNA weakened the proliferation and viability of oral cancer cells. Combined with miR-219-5p inhibitor and SOX5 siRNA partially neutralized the proliferation and migration promotion induced by miR-219-5p inhibitor. Similarly, treatment with both miR-291-5p mimics and SOX5 upregulated plasmids attenuated the suppression in viability and mobility induced by miR-219-5p in oral cancer cells. The present study demonstrated the potential roles of miR-219-5p in the development and progress of oral cancer by targeting SOX5.

The potential roles of SOX5 in various kinds of tumors were available in the database and public literature. Huang et al. found that SOX 5 promoted the progress of nasopharyngeal carcinoma by inducing the down-regulation of SPARC protein expression, and the overexpression of SOX 5 in carcinoma was positively correlated with low clinical survival rate[18]. In cases with liver cancer and breast cancer, SOX5 promotes epithelial mesenchymal transition (EMT) and tumor cells invasion through smad3-SOX5-twist1 signaling pathway $[19,20]$. In prostate cancer, SOX5 regulates the TGF- $\beta$-induced EMT by regulating the expression of Twist1, thus enhanced the metastasis of prostate cancer cells[21]. Currently, researchers present evidences that SOX5 may serve as a tumor metastasis-promoting gene in patients with gastric cancer. Data suggested that patients with high expression of SOX5 were more likely to have metastasis[22]. In addition, the loss of endogenous SOX5 expression attenuates the invasion and metastasis of gastric cancer cells, while overexpression of SOX5 significantly reverses these events[23]. Furthermore, other studies have pointed out that member proteins of SOX family detected in human cancer tissues, were served as potential molecular markers[24]. It is proved that SOX5 is up-regulated in various forms of cancer and is associated with poor prognosis of patients. However, there are rare reports about the expression of SOX5 and its possible roles in the occurrence, development and metastasis of oral cancer. The results of this study demonstrated that the expression of SOX5 increased in oral cancer tissues and the expression levels of SOX5 was positively related with the poor prognosis of patients. Overexpression of SOX5 enhanced the proliferation, migration and invasion of oral cancer cells. By contrast, knockdown of SOX5 expression suppressed the proliferation, migration and invasion of oral 
cancer cells. These results support that SOX5 might served as a promise molecular markers for diagnose and prognosis of patients with oral cancer.

A series studies reveal that miRNAs play essential roles in regulating the proliferation, invasion and metastasis of oral tumors, which is a leading cause of death in patients. Recent studies have found that miR-219-5p inhibited the proliferation, migration and invasion of epidermal ovarian cancer cells by regulating the Twist/Wnt/ $\beta$-catenin signaling pathway[25]. Other studies reported that miR-219-5p was downregulated in gastric cancer, and might serve as a gastric cancer suppressor by inhibiting cell proliferation, migration, and invasion by targeting the LRH-1 and Wnt/ $\beta$-catenin signaling pathways[26]. In colorectal cancer cases, miR-219-5p inhibited the proliferation and invasion process of cancer cells by down-regulating the expression of Calcyphosin and PDGF receptor[27,28], and was proved to inhibit the EMT and metastasis process by down-regulating the expression of LEBF1[29]. However, the expression changes and possible roles of miR-219-5p in oral cancer are still unclear. In this study, the expression of miR-219-5p in oral cancer tissues and cells were determined. The results showed that the expression level of miR-219-5p in oral cancer tissues was significantly lower than that in adjacent tissues. Moreover, we demonstrated that miR-219-5p mimics inhibited the proliferation, migration and invasion of oral cancer cells. Conversely, inhibition of miR-219-5p expression promoted the growth and invasion of oral cancer cells. The present data demonstrated a novel role of miR-219-5p as a tumor suppressor in oral cancer.

Followed, this study determined whether miR-291-5p regulated the proliferation, migration and invasion of oral cancer cells by targeting SOX5. Databases prediction and luciferase reporter assays verified SOX5 as one of the target gene of miR-219-5p by interacting with the 3 '- UTR region of SOX5 mRNA. Treatment with miR-291-5p mimics or inhibitors induced significant decrease or increase in SOX5 expression levels, respectively. Combined with miR-219-5p inhibitor and SOX5 siRNA partially neutralized the proliferation and migration promotion induced by miR-219-5p inhibitor. Treatment with both miR-291-5p mimics and SOX 5 up-regulated plasmids attenuated the suppression in viability and mobility induced by miR-219-5p in oral cancer cells. Given those, this study demonstrated that miR-291-5p inhibited the proliferation, migration and invasion of oral cancer cells by targeting SOX5. It was noticed that transfection with SOX5 siRNA or pcDNA3.1-SOX5 leaded to partial, rather than rounded, neutralization of the corresponding effects induced by miR-219-5p mimics or inhibitor treatment. It is well demonstrated that a single miRNA species, in association with Argonaute proteins, may target hundreds or even thousands of different mRNAs[30,31]. Therefore, miR-291-5p may be involved in the progress of oral cancer through other targets regulating, besides SOX5. However, further investigation is necessary.

This study indicates novel roles of miR-219-5p in the proliferation, migration and invasion of oral cancer cells, and demonstrates its mechanism by regulating the target gene SOX5. This work will provide evidences for novel biomarker for dignosis and prognosis of patients with oral cancer and supply promise drug target for clinical therapy.

\section{Declarations}


Acknowledgements

We thank all those who participated in this study.

\section{Authors' contributions}

Conceptualization: TZ, YB and RM; Methodology: TZ, YH, XH, BC, JT, RM; Formal analysis and investigation: $\mathrm{BC}$, JT and $\mathrm{YB}$; Writing - original draft preparation: $\mathrm{XH}$; Writing - review and editing: $\mathrm{YB}$ and RM; Funding acquisition: RM; Resources: RM, BC, JT; Supervision: YB.

\section{Funding}

This work was supported by the Scientific research Foundation of Ministry of Education, Yunnan Province, China (Grant Nos. 2019J1292).

Availability of data and materials

Not applicable

\section{Ethics approval and consent to participate}

Not applicable

Consent for publication

Not applicable

Competing interests

The authors declare that they have no competing interests

Footnotes

\section{Publisher's Note}

Springer Nature remains neutral with regard to jurisdictional claims in published maps and institutional affiliations.

\section{Contributor information}

Ting Zhou,E-mail: 1337882363@qq.com

Ying He,E-mail: 1505115263@qq.com

Xiao-Han Dong,E-mail:xiaohan13460@126.com

Yan-Bin Xiyang,E-mail: xiyang_neuro@126.com 
Bo Chen,E-mail: chenbo@kmmu.edu.cn

Jun Ton,E-mail:526783703@qq.com

Rui Mao,E-mail: maorui@kmmu.edu.cn

\section{References}

1. Tandon P,Dadhich A, Saluja H, Bawane S, Sachdeva S. The prevalence of squamous cell carcinoma in different sites of oral cavity at our Rural Health Care Centre in Loni, Maharashtra - a retrospective 10-year study. Contemporary Oncology.2017;21(2): 178-183.

2. Wahid F, Shehzad A, Khan T, Kim YY. MicroRNAs: synthesis, mechanism, function, and recent clinical trials. Biochim Biophys Acta.2010;1803(11): 1231-43.

3. Macfarlane LA, Murphy PR. MicroRNA: Biogenesis, Function and Role in Cancer. Curr Genomics.2010;11(7): 537-61.

4. Iorio MV, Croce CM. microRNA involvement in human cancer. Carcinogenesis.2012;33(6): 1126-1133.

5. Long J, Menggen Q, Wuren Q, Shi Q, Pi X. MiR-219-5p Inhibits the Growth and Metastasis of Malignant Melanoma by Targeting BCL-2. Biomed Res Int.2017;2017: 9032502.

6. Yang J, Sheng YY, Wei JW, Gao XM, Zhu Y, Jia HL, Dong QZ, Qin LX. MicroRNA-219-5p Promotes Tumor Growth and Metastasis of Hepatocellular Carcinoma by Regulating Cadherin 1. Biomed Res Int.2018;2018: 4793971.

7. Nititham J, Gupta R, Zeng X, Hartogensis W, Nixon DF, Deeks SG, Hecht FM, Liao W. Psoriasis risk SNPs and their association with HIV-1 control, Hum Immunol.2017;78(2): 179-184.

8. Hardin M, Cho M, McDonald ML, Beaty T, Ramsdell J, Bhatt S, van Beek EJ, Make BJ, Crapo JD, Silverman EK, Hersh CP. The clinical and genetic features of COPD-asthma overlap syndrome. Eur Respir J.2014;44(2): 341-50.

9. Huang DY, Lin YT, Jan PS, Hwang YC, Liang ST, Peng Y, Huang CY, Wu HC, Lin CT. Transcription factor SOX-5 enhances nasopharyngeal carcinoma progression by down-regulating SPARC gene expression. J Pathol.2008;214(4): 445-55.

10. Renjie W, Haiqian L. MiR-132, miR-15a and miR-16 synergistically inhibit pituitary tumor cell proliferation, invasion and migration by targeting Sox5. Cancer Letters.2015;356(2, Part B): 568-578.

11. Wang D, Han S, Wang X, Peng R, Li X. SOX5 promotes epithelial-mesenchymal transition and cell invasion via regulation of Twist1 in hepatocellular carcinoma. Med Oncol.2015;32(2): 461.

12. Pei XH, Lv XQ, Li HX. Sox5 induces epithelial to mesenchymal transition by transactivation of Twist1. Biochemical and Biophysical Research Communications.2014;446(1): 322-327.

13. Hu J, Tian J, Zhu S, Sun L, Yu J, Tian H, Dong Q, Luo Q, Jiang N, Niu Y, Shang Z. SOX5 contributes to prostate cancer metastasis and is a master regulator of TGF- $\beta$-induced epithelial mesenchymal transition through controlling Twist1 expression. British Journal Of Cancer.2017;118(1): 88-97. 
14. Lifang Wang, Shucheng Ye, Junye Wang, Zhenfang Gu, Yanhui Zhang, Chunmei Zhang, Xuezhen Ma. HuR Stabilizes Inc-Sox 5 mRNA to Promote Tongue Carcinogenesis.Biochemistry (Mosc).2017;82(4):438-445.

15. Song SW, Fajol A, Tu XK, Ren BG, Shi SS. miR-204 suppresses the development and progression of humanglioblastoma by targeting ATF2. Oncotarget.2016;7(43):70058-70065.

16. Livak KJ, Schmittgen TD. Analysis of relative gene expression data using real-time quantitative PCR and the 2(-Delta Delta C(T)) method. Methods.2001;25(4): 402-408.

17. He Z, Xia Y, Liu B, et al. Down-regulation of miR-452 is associated with poor prognosis in the nonsmall-cell lung cancer. J ThoracDis.2016;8:894-900.

18. Huang DY, Lin YT, Jan PS, Hwang YC, Liang ST, Peng Y, Huang CY, Wu HC, Lin CT. Transcription factor SOX-5 enhances nasopharyngeal carcinoma progression by down-regulating SPARC gene expression. J Pathol.2008;214(4): 445-55.

19. Wang D, Han S, Wang X, Peng R, Li X. SOX5 promotes epithelial-mesenchymal transition and cell invasion via regulation of Twist1 in hepatocellular carcinoma. Med Oncol.2015;32(2): 461.

20. Pei XH, Lv XQ, Li HX. Sox 5 induces epithelial to mesenchymal transition by transactivation of Twist1. Biochemical and Biophysical Research Communications.2014;446(1): 322-327.

21. Hu J, Tian J, Zhu S, Sun L, Yu J, Tian H, Dong Q, Luo Q, Jiang N, Niu Y, Shang Z. Sox 5 contributes to prostate cancer metastasis and is a master regulator of TGF- $\beta$-induced epithelial mesenchymal transition through controlling Twist1 expression. British Journal Of Cancer.2018;118(1): 88-97.

22. Shi Ding ,Yanping Zhang. MicroRNA-539 inhibits the proliferation and migration of gastric cancer cells by targeting SRY-box 5 gene.Mol Med Rep.2019;20(3): 2533-2540.

23. Jianxiong You, Qing Zhao, Xindong Fan, and Jingbing Wang. SOX5 promotes cellinvasion and metastasis via activation of Twist-mediated epithelial-mesenchymal transition in gastric cancer. Onco Targets Ther.2019;12: 2465-2476.

24. Apiwat Pugongchai, Andrey Bychkov, Pichet Sampatanukul. Promoter hypermethylation of SOX11 correlates with adverse clinicopathological features of human prostate cancer. Int J Exp Pathol.2017;98(6): 341-346.

25. Wei C, Zhang X, He S, Liu B, Han H, Sun X. MicroRNA-219-5p inhibits the proliferation, migration, and invasion of epithelial ovarian cancer cells by targeting the Twist/Wnt/ $\beta$-catenin signaling pathway. Gene.2017;637: 25-32.

26. Li C, Dong J, Han Z, Zhang K.MicroRNA-219-5p Represses the Proliferation, Migration, and Invasion of Gastric Cancer Cells by Targeting the LRH-1/Wnt/beta-Catenin Signaling Pathway. Oncol Res.2017;25(4): 617-627.

27. Wang Q, Zhu L, Jiang $Y, X u$ J, Wang F, He Z. miR-219-5p suppresses the proliferation and invasion of colorectal cancer cells by targeting calcyphosin. Oncol Lett.2017;13(3): 1319-1324.

28. Xiong GB, Zhang GN, Xiao Y, Niu BZ, Qiu HZ, Wu B, Lin GL, You L, Shu H. MicroRNA-219-5p functions as a tumor suppressor partially by targeting platelet-derived growth factor receptor alpha in colorectal cancer. Neoplasma.2015;62(6): 855-63. 
29. Huang LX, Hu CY, Jing L, Wang MC, Xu M, Wang J, Wang Y, Nan KJ, Wang SH.microRNA-219-5p inhibits epithelial-mesenchymal transition and metastasis of colorectal cancer by targeting lymphoid enhancer-binding factor 1. Cancer Sci.2017;108(10): 1985-1995.

30. Baek D, Villen J, Shin C, Camargo FD, Gygi SP, Bartel DP. The impact of microRNAs on protein output. Nature.2008;455(7209):64-71.

31. Miranda KC, Huynh T, Tay Y, Ang YS, Tam WL, Thomson AM, Lim B, Rigoutsos I.A pattern-based method for the identification of MicroRNA binding sites and their corresponding heteroduplexes. Cell.2006;126(6):1203-1217.

\section{Figures}



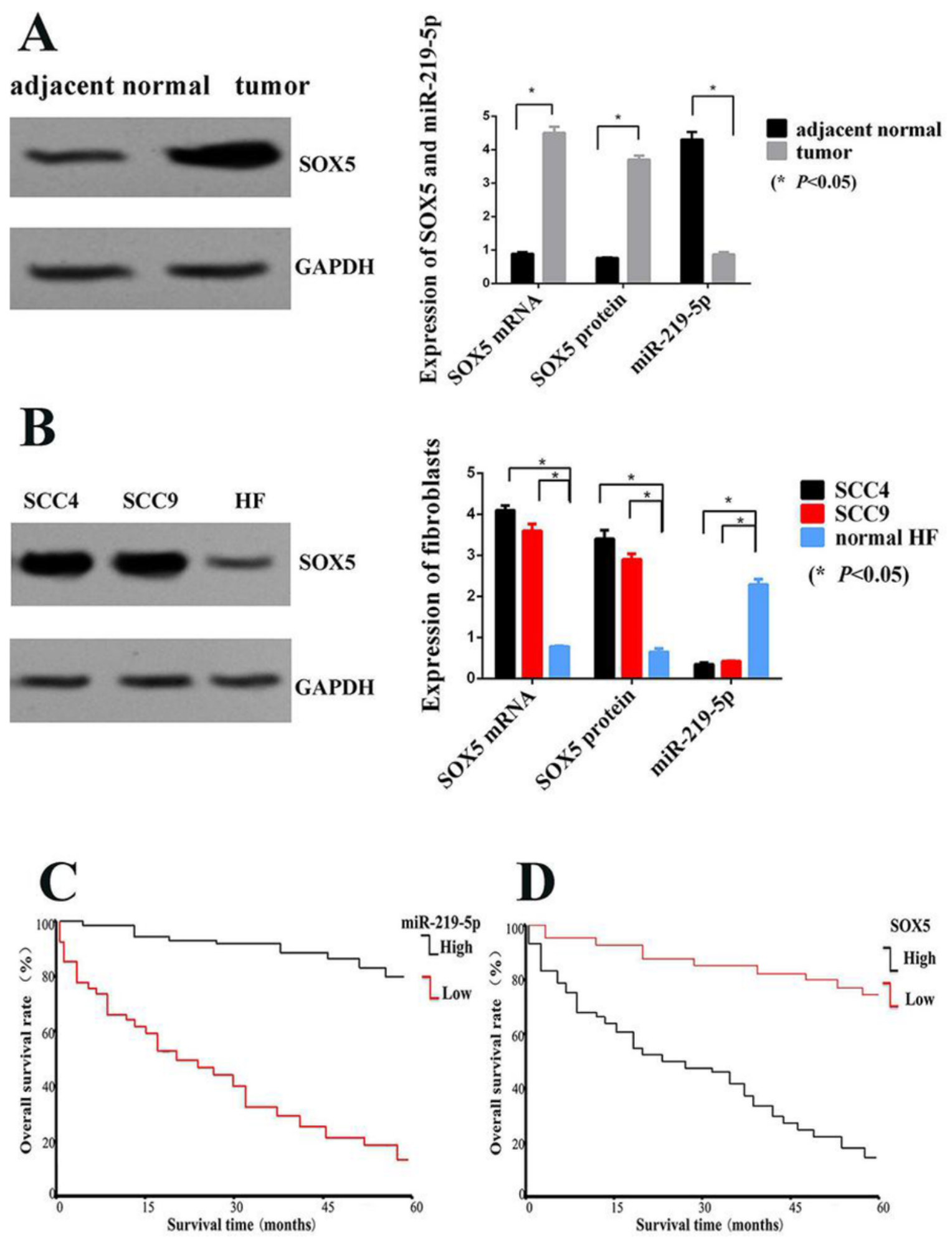

\section{Figure 1}

Abnormal expression levels of miR-219-5p and SOX5 in tumor tissues and cell lines of oral cancer (a)The relative expression of SOX5 and miR-219-5p in oral cancer and adjacent normal tissues indicated by Western blot and/or RT-qPCR analysis; (b) The relative expression levels of SOX5 and miR-219-5p in oral cancer cells and normal HF cell lines detected by Western blot and/or RT-qPCR analysis. Compared with normal HF and tongue squamous cell carcinoma cells, the expression levels of SOX5 were increased and 
the expression level of miR-219-5p was significantly decreased $(P<0.05)$. (c)The relationship between miR-219-5p expression level and survival rate of patients. (d)The relationship between expression level of SOX5 and survival rate of patients.
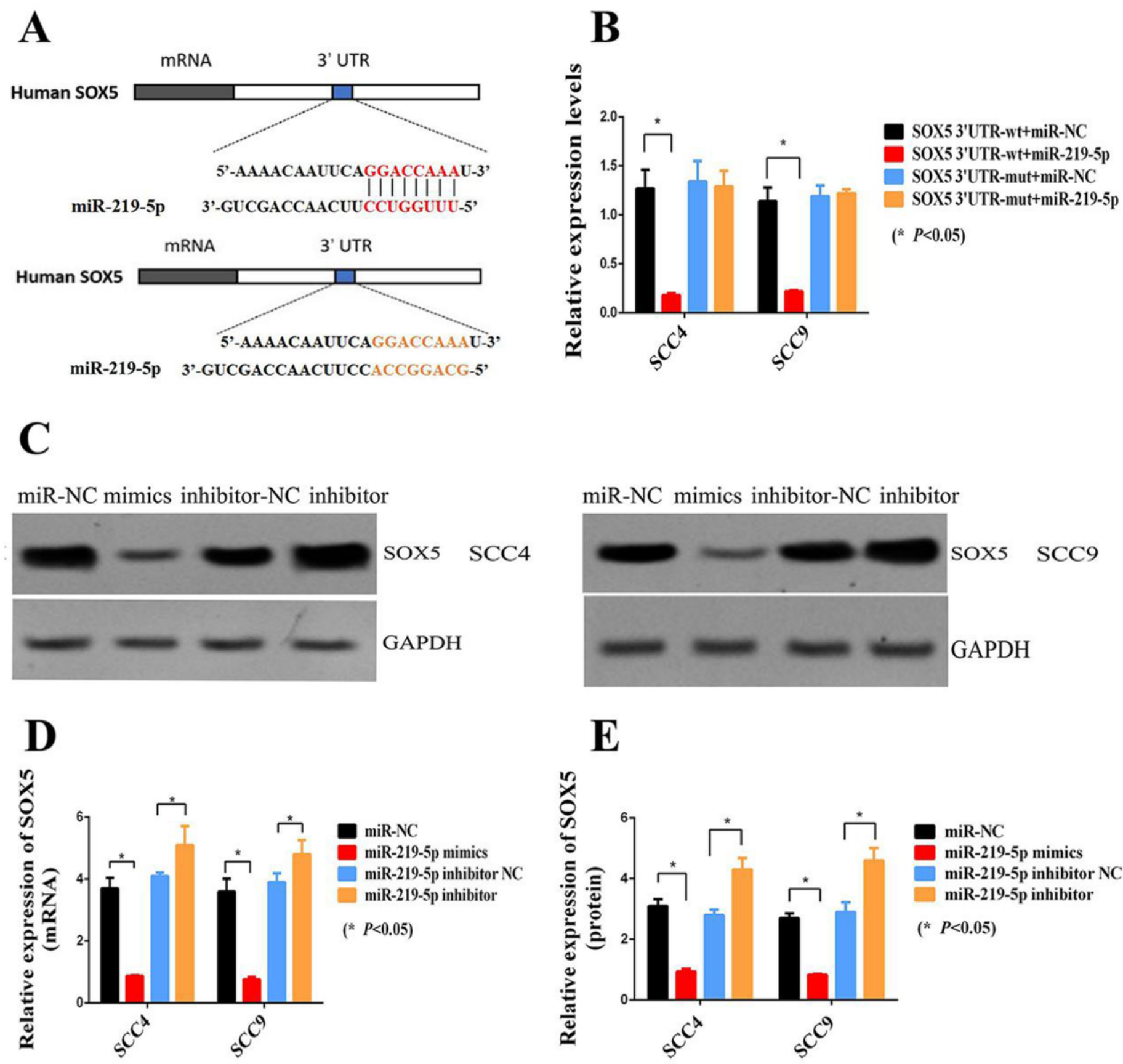

miR-NC mimics inhibitor-NC inhibitor
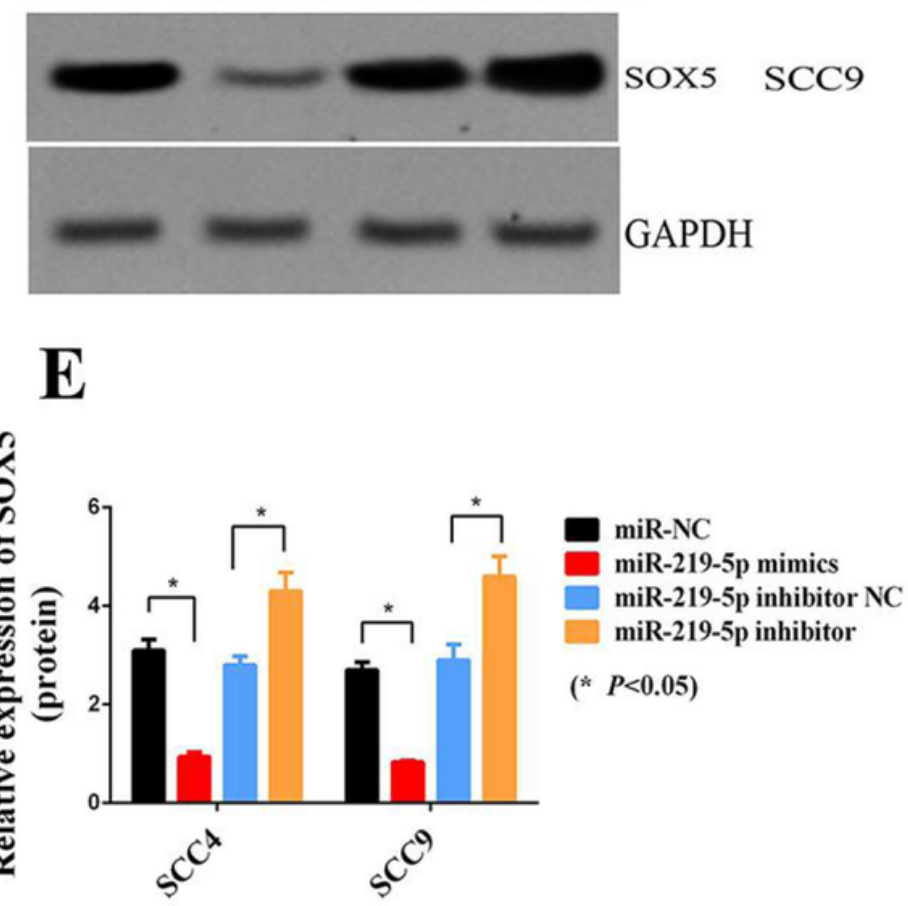

\section{Figure 2}

miR-219-5p targets SOX5 by binding to the 3'-UTR of SOX5 mRNA (a):Prediction of the target genes of miR-219-5p using online miRNA target prediction databases. Based on the list of the putative miR-219-5p binding sequences in the 3'-UTR of SOX5, mutations were generated. The mutated sequences were indicated in the schematic diagram. (b):The wild-type (wt) and mutant (mut) plasmids of the 3 '-UTR of SOX5 mRNA were cloned, and the wt- or mut- sequences were inserted into the pmirGlo vector by 
molecular cloning. Then the relative activity of luciferase was detected $48 \mathrm{~h}$ after co-transfection with miR-219-5p mimics /miR-219-5p negative control and PhRM-TK Renilla reporter vector. Western blot (c) and RT-qPCR (d, e) were performed to evaluate the expression levels of SOX5 in SCC4 and SCC9 cells treated with miR-219-5p mimics or inhibitor. NC: negative control

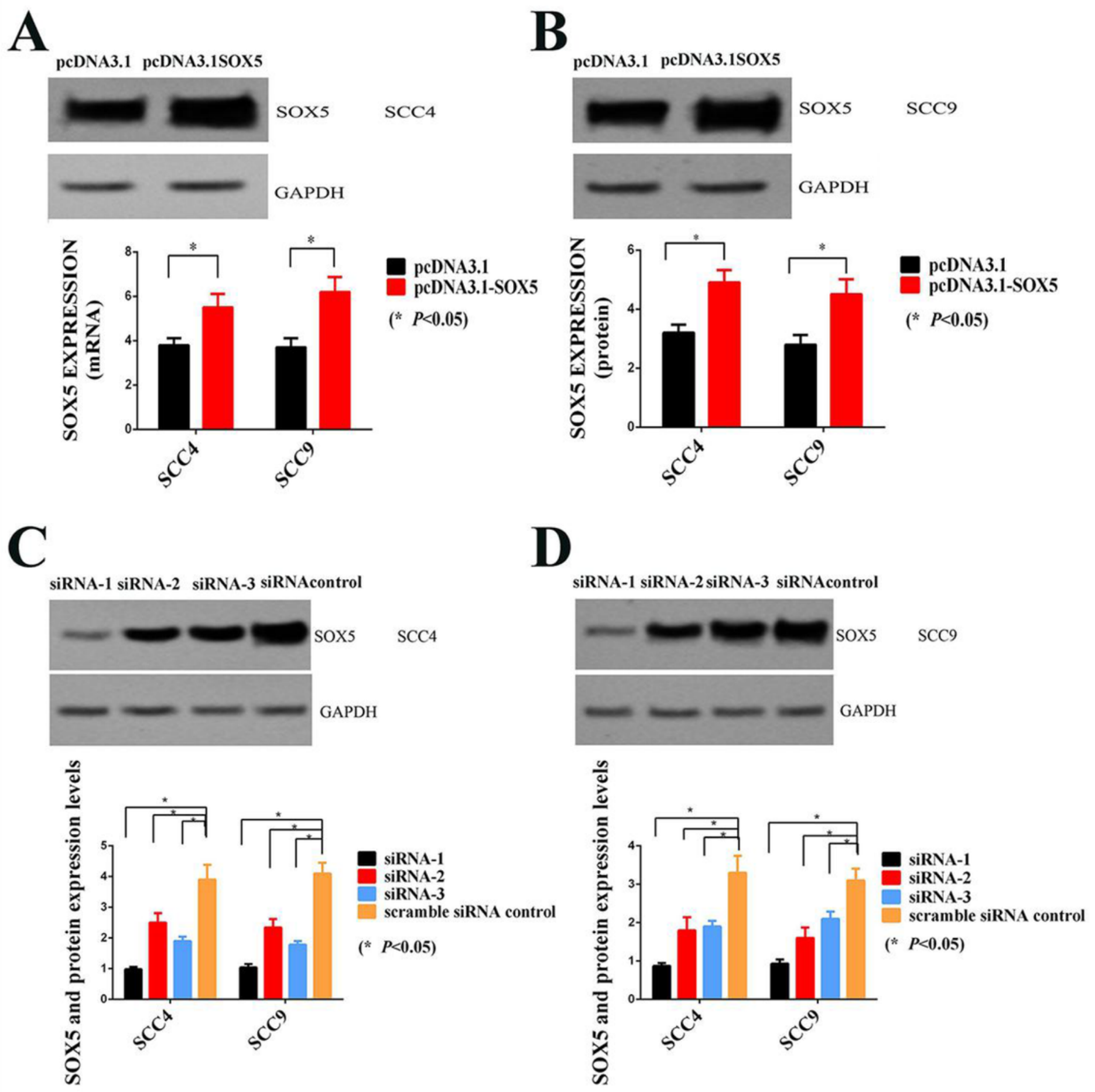

Figure 3

Evaluation the transfection efficiency of pcDNA3.1-SOX5 and siRNAs introduction in SCC4 and SCC9 cells (a):Western blot and RT-qPCR were performed to evaluate the expression levels of SOX5 in SCC4 
and SCC9 cells treated with pcDNA3.1-SOX5.(b):Western blot and RT-qPCR were performed to evaluate the expression levels of SOX5 in SCC4 and SCC9 cells treated with pCDNA3.1-SOX5.(c):Western blot and RT-qPCR were performed to evaluate the expression levels of SOX 5 to determine the siRNA interference efficiency.(d):Western blot and RT-qPCR were performed to evaluate the expression levels of SOX5 to determine the siRNA interference efficiency.(Fig.3. P<0.05)

\section{A}

pcDNA3.1 pcDNA3.1-SOX5 siRNA control SOX5-siRNA
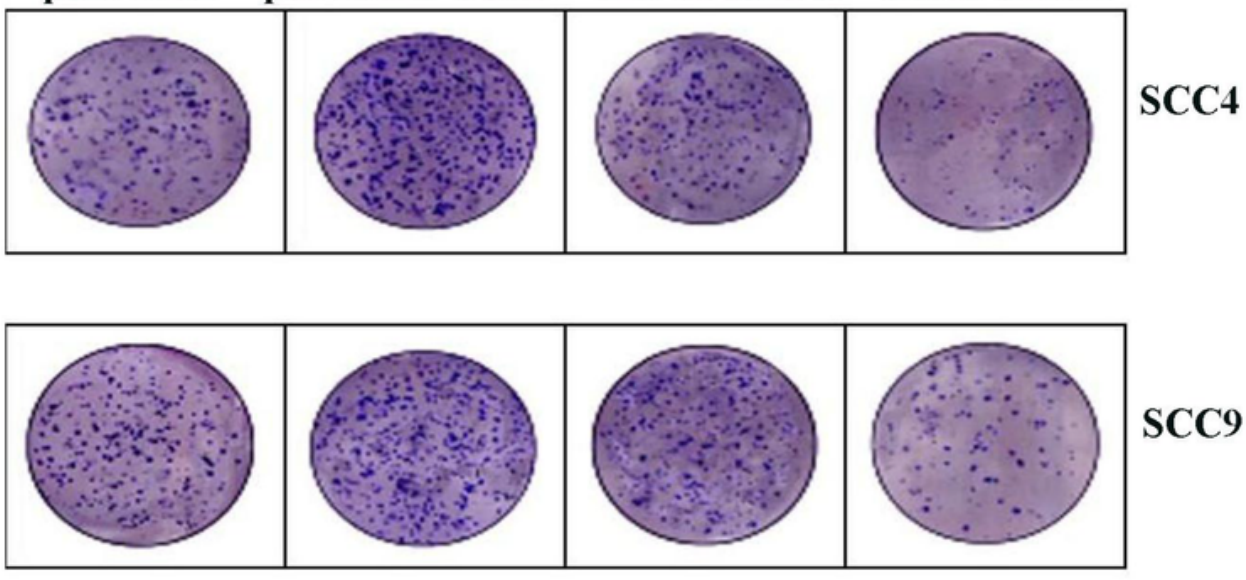

B

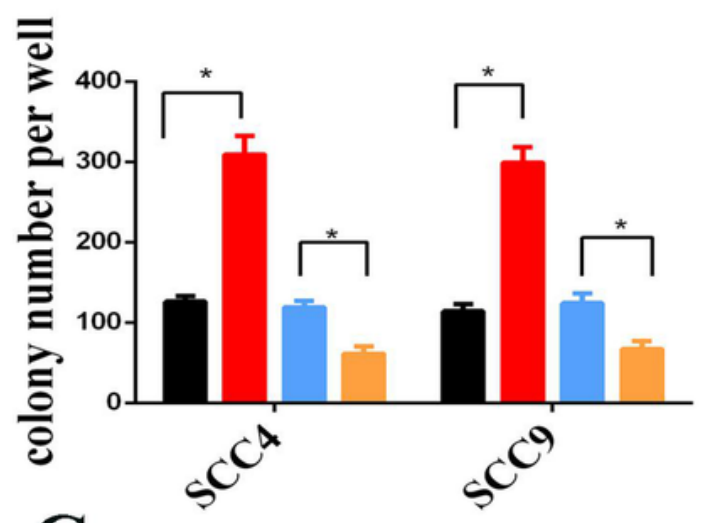

C

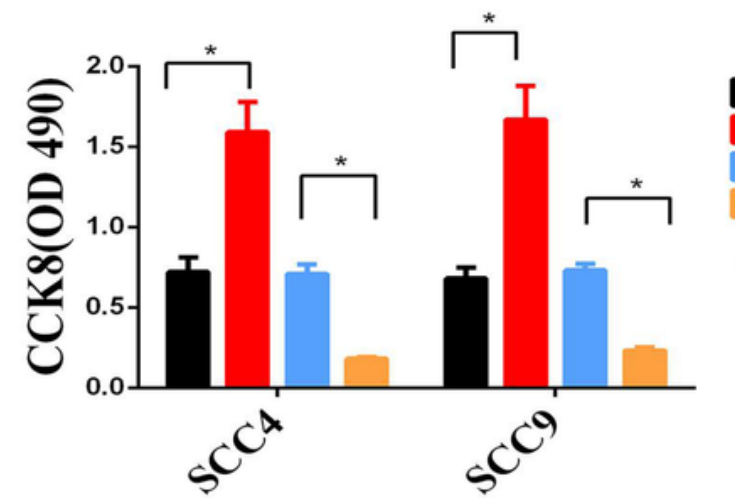

pcDNA3.1

pcDNA3.1-SOX5

scramble siRNA control SiRNA-SOX 5

(* $P<0.05)$

Figure 4 
SOX5 expressional regulation affects the vitality of oral cancer cells (a):Representative micrographs of colony formation. Colony formation (b) and CCK-8 assays (c) were used to detect the expression levels of SOX5 overexpressed and under-expressed cells. Magnification, 100X

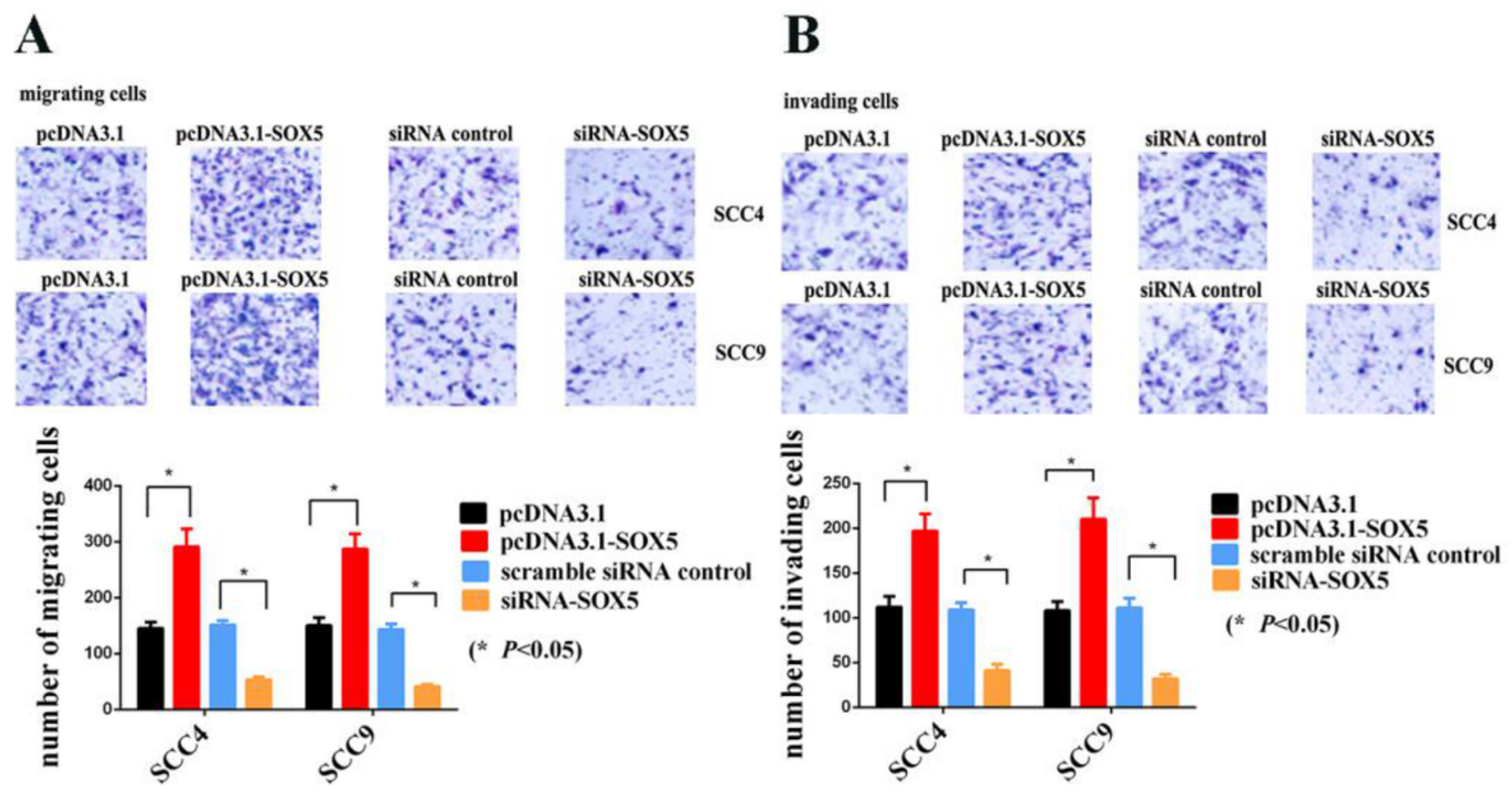

Figure 5

SOX5 alters the migration and invasion of oral cancer cells Cell migration (a) and invasion (b) were evaluated following treatment with SOX5 overexpression (pcDNA3.1-SOX5) or inhibition (siRNA-SOX5), respectively. The representative crystal violet stained cells from different groups were exhibited.

Magnification, 200X 


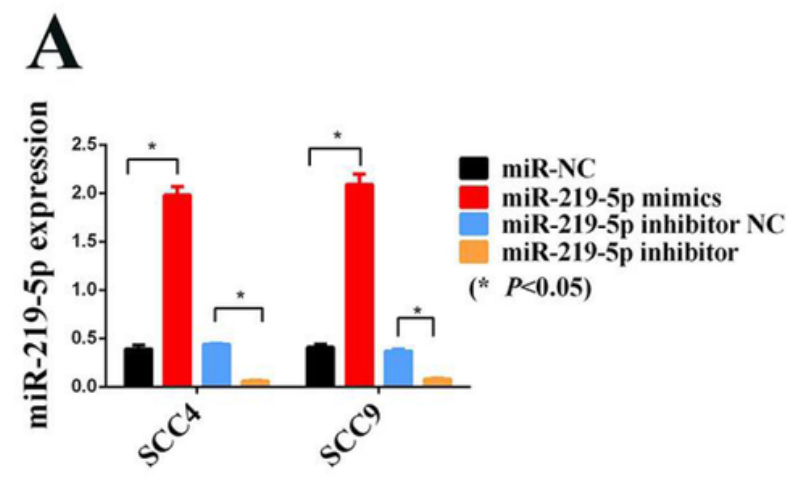

B
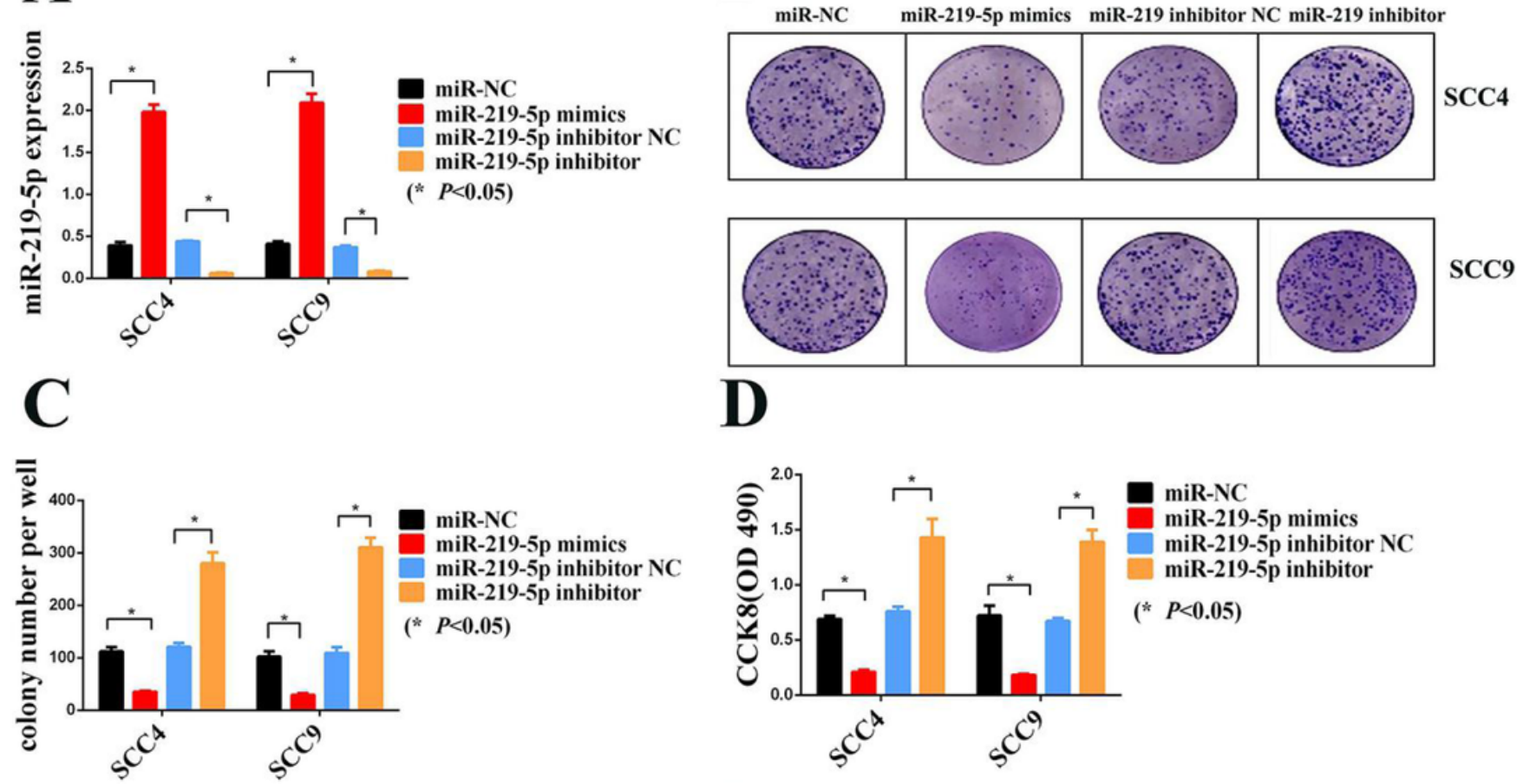

D
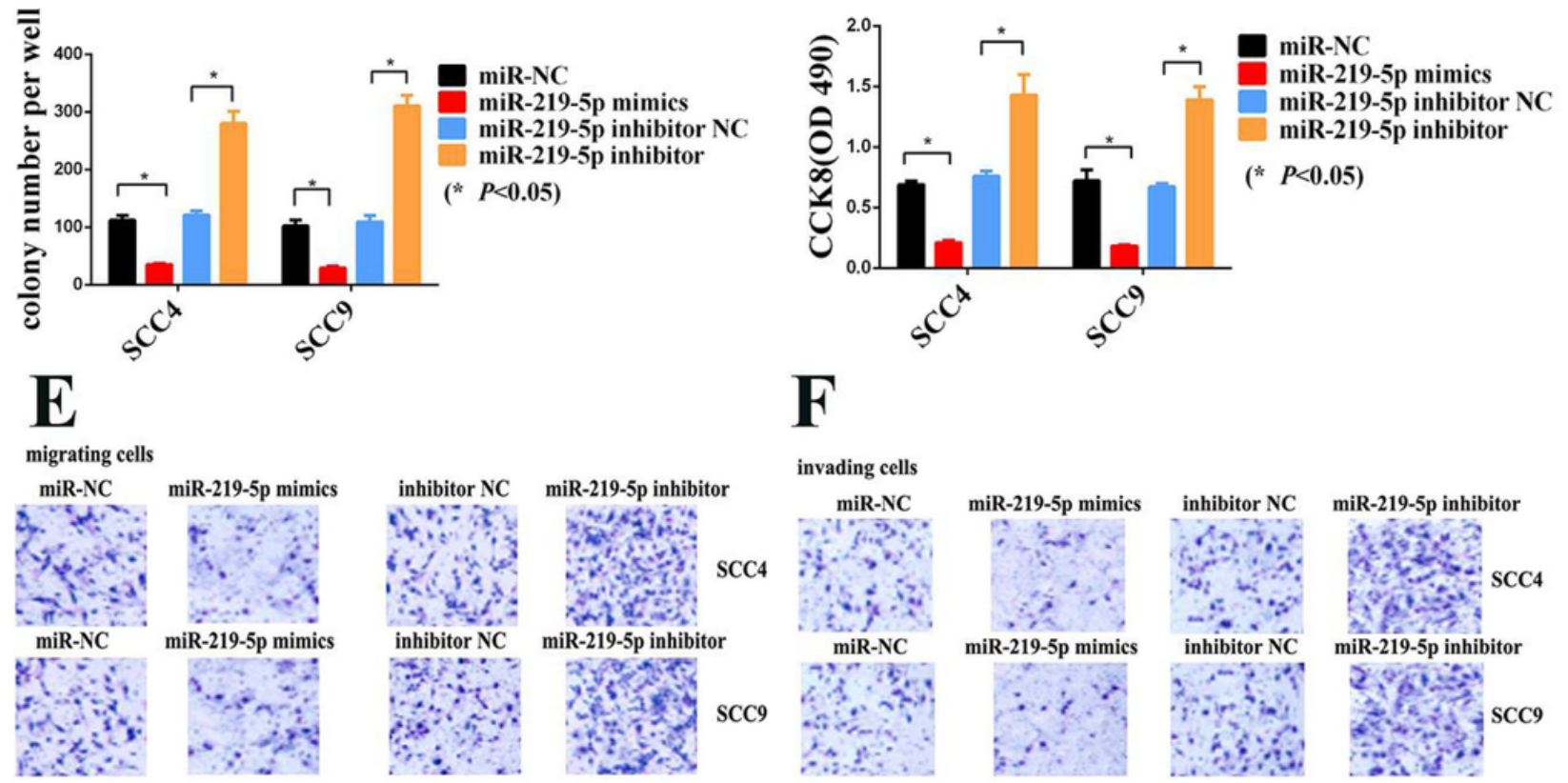

\section{F}
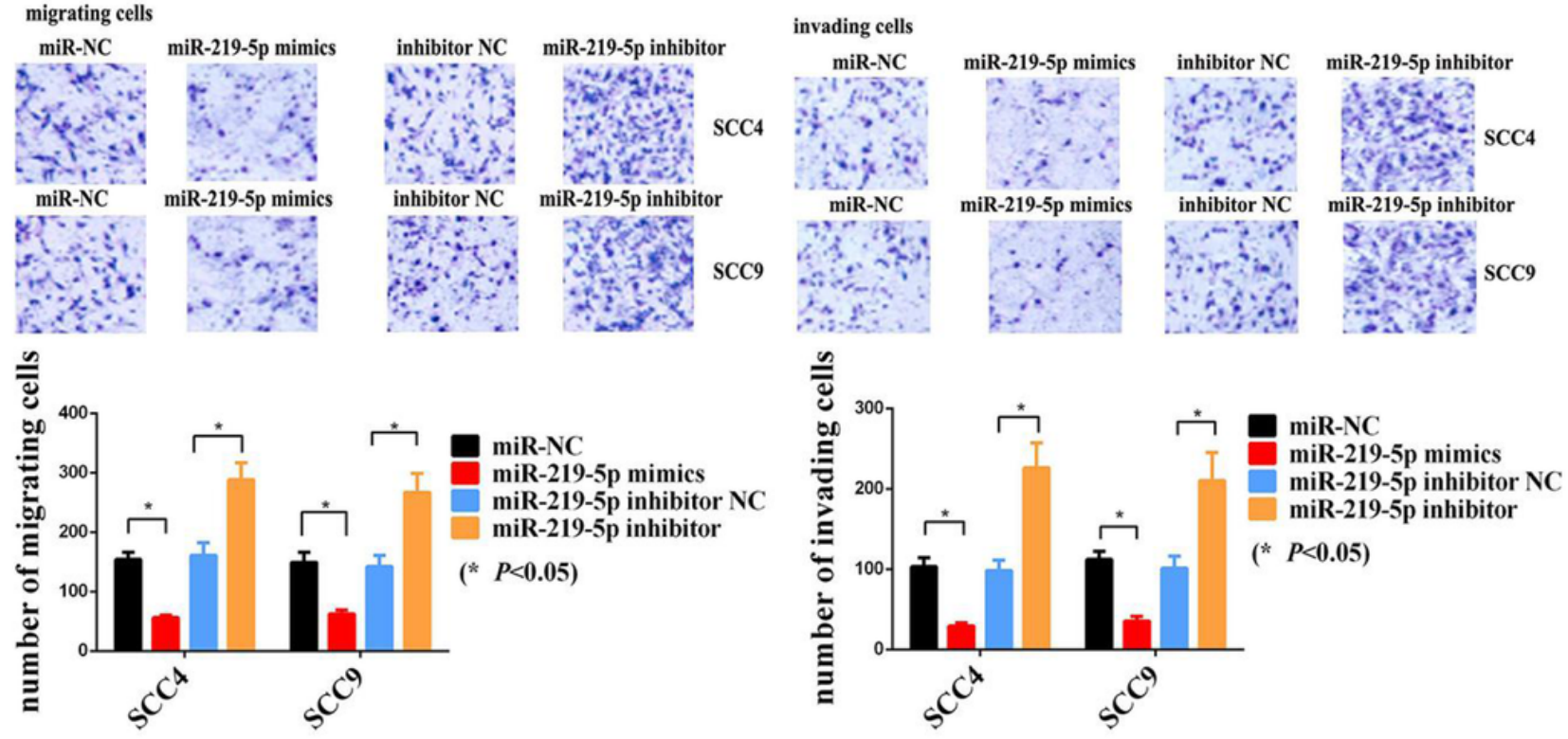

Figure 6

miR-219-5p weakens the viability and mobility of oral cancer cells (a):The miR-219-5p levels were detected in SCC4 and SCC9 cells treated with miR-219-5p mimics or inhibitor by RT-qPCR.

(b):Representative micrographs of colony formation assays from SCC4 and SCC9 cells treated with miR219-5p mimics or inhibitor. Magnification, 100X. The quantitative analysis of the colony formation (c) and CCK-8 assays (d) were performed. Cell migration (e) and invasion (f) of SCC4 and SCC9 cells were determined using the Transwell assay. Magnification, 200X. NC: negative control. 
A
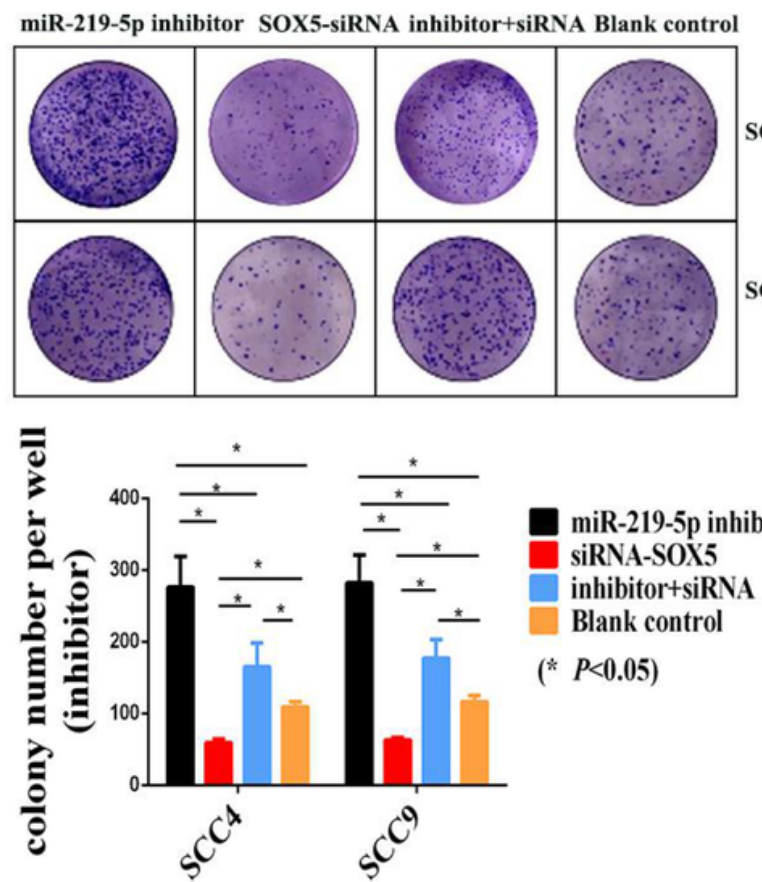

C

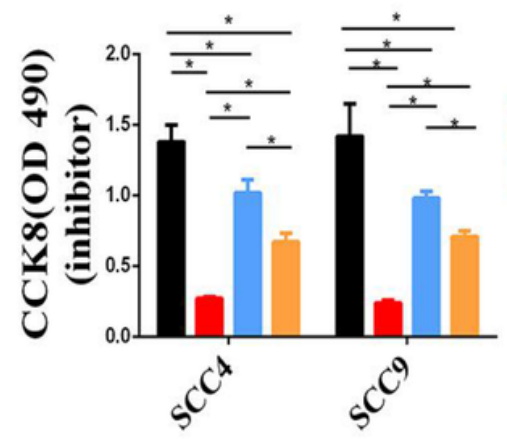

B

miR-219-5p mimics pcDNA3.1-SOX5 mimics+SOX5 Blank control
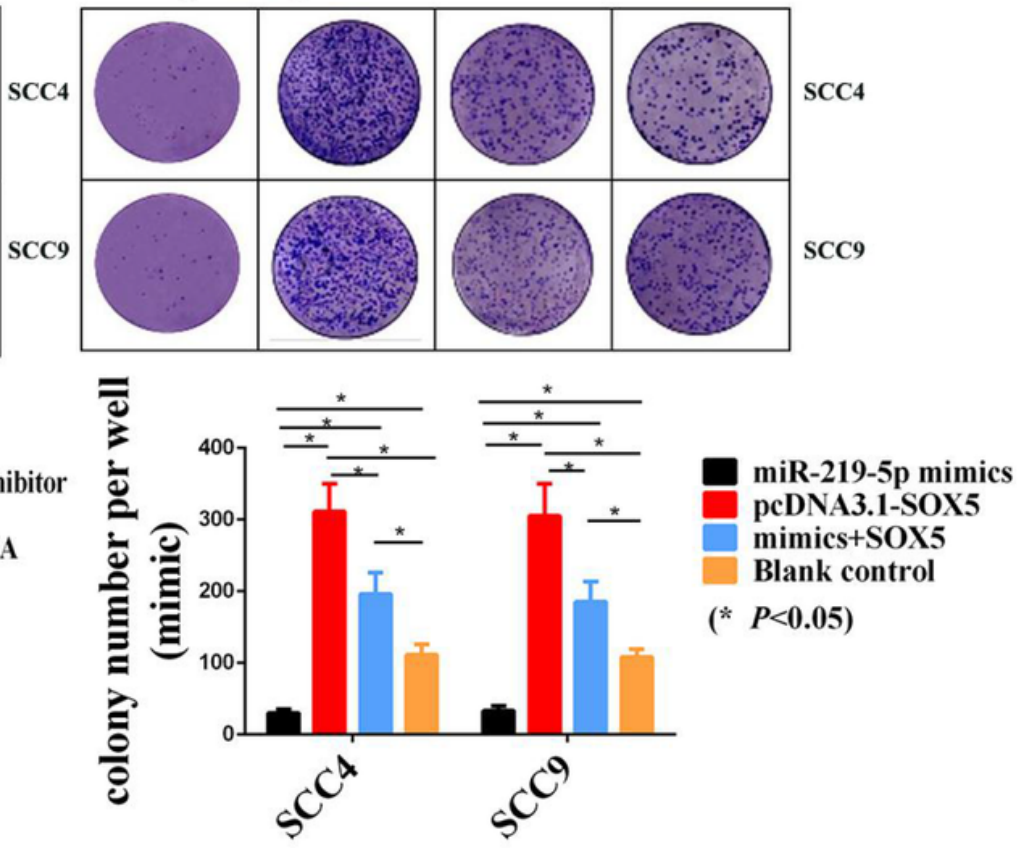

D

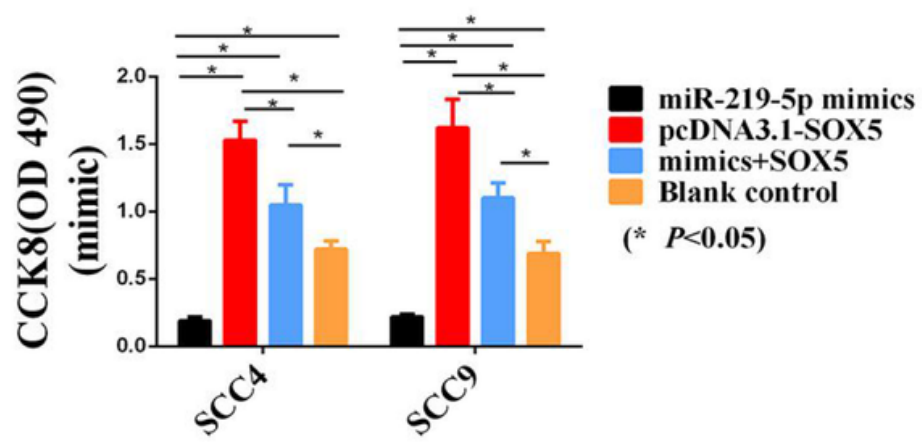

Figure 7

Effects of miR-219-5p/SOX5 on the viability of oral cancer cells The representative stained colonies and quantitative analysis of colony formation $(a, b)$ and CCK-8 assays $(c, d)$ were performed to evaluate whether the effects of miR-219-5p on the cell viability were depended on regulating SOX5. SCC 4 and SCC9 cells were treated with miR-219-5p inhibitor or/and siRNA-SOX5, AS as well as miR-219-5p mimics or/and pcDNA3.1-SOX5. The innocent cells were served as blank control. Magnification, 100X. 

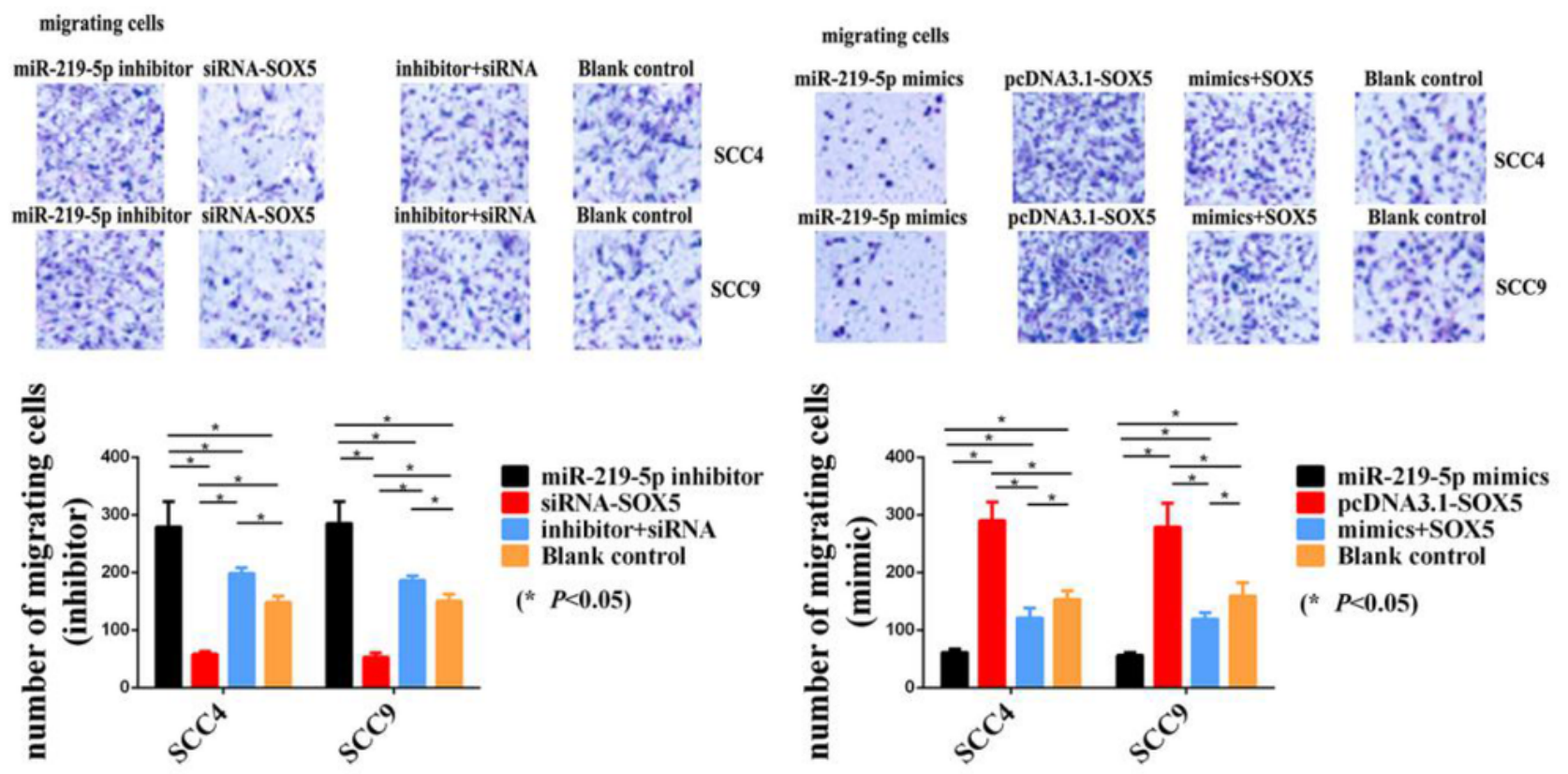

\section{B}

invading cells

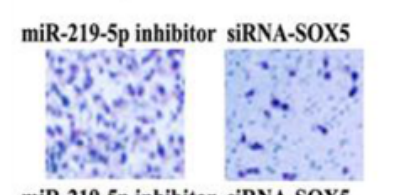

miR-219-5p inhibitor siRNA-SOX5
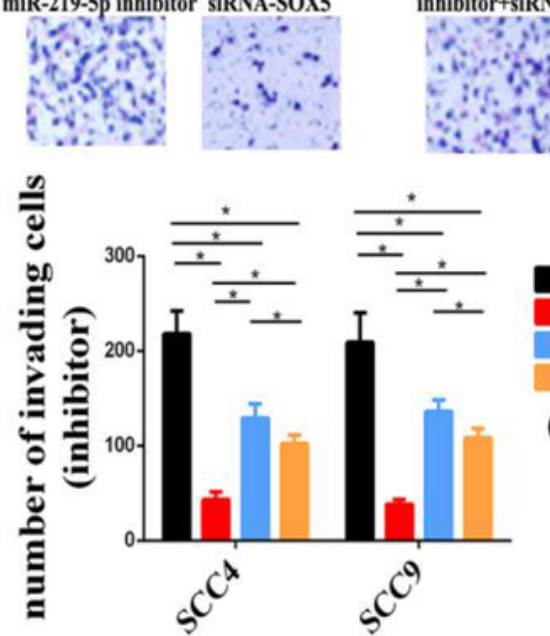

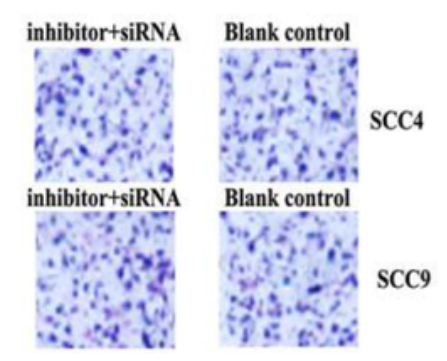

miR-219-5p inhibitor siRNA-SOX5 inhibitor+siRNA Blank control

(* $P<0.05$ )
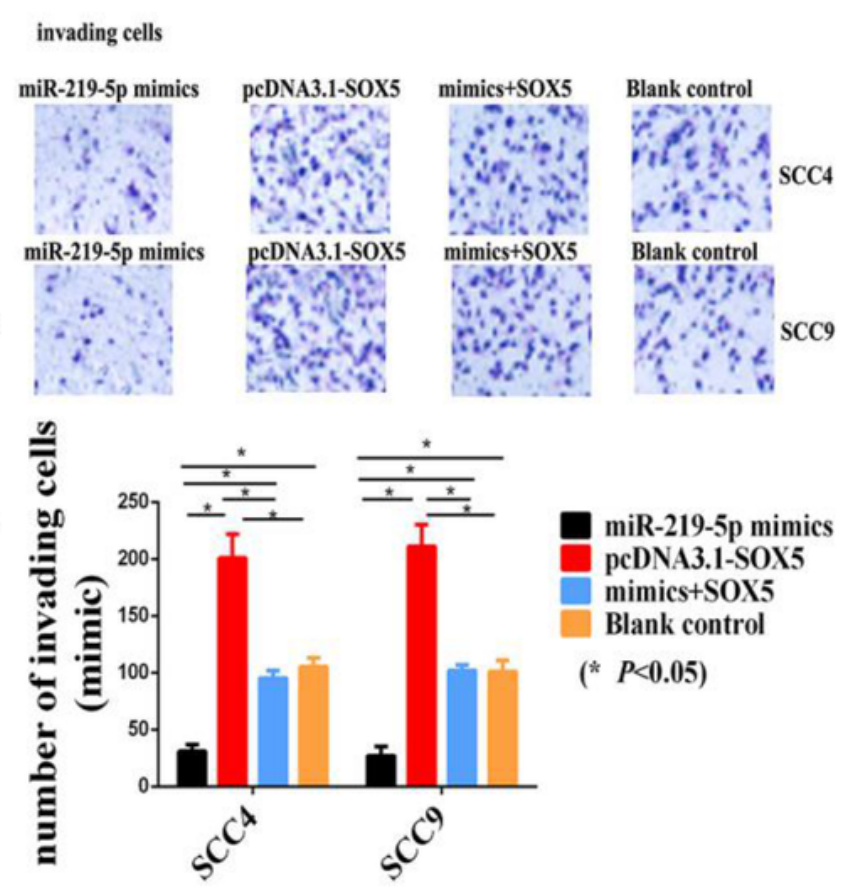

\section{Figure 8}

Effects of miR-219-5p/SOX5 on the mobility of oral cancer cells The representative stained cells and quantitative analysis of migration assays (a) and invasion assays (b) were performed to evaluate whether the effects of miR-219-5p on the cell mobility were depended on targetingSOX5. SCC4 and SCC9 cells were treated with miR-219-5p inhibitor or/and siRNA-SOX5, as well as well as miR-219-5p mimics or/and pcDNA3.1-SOX5. The innocent cells were served as blank control. Magnification, 200X. 